\title{
Improved Ratio Estimators for the Population Mean Using Non-Conventional Measures of Dispersion
}

\author{
Muhammad Abid \\ Department of Mathematics \\ Institute of Statistics \\ Zhejiang University, Hangzhou, 310027, China \\ Nasir Abbas \\ Department of Statistics \\ Faculty of Science and Technology \\ Government College University, Faisalabad, Pakistan \\ Rehan Ahmad Khan Sherwani \\ College of Statistical and Actuarial Sciences \\ University of the Punjab Lahore, Pakistan \\ rehan.stat@pu.edu.pk \\ Hafiz Zafar Nazir \\ Department of Statistics \\ Faculty of Science \\ University of Sargodha, Pakistan
}

\begin{abstract}
In recent times, it is common to the make use of auxiliary information to increase the precision of estimators in sample surveys. In this study, we propose some new modified linear regression type ratio estimators for estimating population mean by some non-conventional dispersion measures such as: Gini's mean difference, Downton's method and probability weighted moments with linear combination of population correlation coefficient and population coefficient of variation. Expressions for the bias and the mean squared error are derived and are compared with those of the usual ratio estimator and the existing ratio type estimators in literature. Conditions are determined for which the proposed estimators perform better than the existing estimators. Both theoretical and empirical findings show the soundness of the proposed procedure for estimation of population mean.
\end{abstract}

Keywords: Auxiliary variable, Downton's technique, Gini's mean difference, Probability weighted moments.

\section{Introduction}

In survey research, there are situations when the information, on every unit in the population, is available. If a variable, that is known for every unit of the population and is not a variable of direct interest but instead employed to improve the sampling plan or to enhance the estimation of the variables of interest, is called an auxiliary variable. The auxiliary information is commonly associated with the use of ratio type estimation methods and to improve the efficiency of the estimators in survey sampling.

Consider a finite population $U=\left\{U_{1}, U_{2}, U_{3}, \ldots, U_{N}\right\}$ of $N$ distinct and identifiable units. Let $Y$ be the study variable with value $Y_{i}$ measured of $U_{i}, i=1,2, \ldots, N$ giving a 
vector $Y=\left\{Y_{1}, Y_{2}, Y_{3}, \ldots, Y_{N}\right\}$. The objective is to estimate population mean $\bar{Y}=\frac{1}{N} \sum_{i=1}^{N} Y_{i}$ on the basis of a random sample. When the population parameters of the auxiliary variable, such as population mean, kurtosis, skewness, coefficient of variation, median, quartiles, correlation coefficient, deciles etc., are known, ratio estimators and their modifications are available in the literature which perform better than the usual sample mean under the simple random sampling without replacement (SRSWOR).

The notations used in this paper can be described as follows:

\section{NOMENCLATURE}

\section{Romen}

$\begin{array}{ll}N & \text { Population size } \\ n & \text { Sample size } \\ f=n /_{N} & \text { Sampling fraction } \\ Y & \text { Study variable } \\ X & \text { Auxiliary variable } \\ \bar{X}, \bar{Y} & \text { Population means } \\ \bar{x}, \bar{y} & \text { Sample means } \\ x, y & \text { Sample totals } \\ S_{x}, S_{y} & \text { Population standard deviations } \\ S_{x y} & \text { Population covariance between } X \text { and } Y \\ C_{x}, C_{y} & \text { Coefficient of variation } \\ B(.) & \text { Bias of the Estimator } \\ M S E(.) & \text { Mean square error of the estimator } \\ \hat{Y}_{i} & \text { Existing modified ratio estimator of } \bar{Y} \\ \hat{\bar{Y}}_{p j} & \text { Proposed modified ratio estimator of } \bar{Y} \\ M_{d} & \text { Median of } X \\ Q D=\frac{Q_{3}-Q_{1}}{2} & \text { Quartile Deviation } \\ G=\frac{4}{N-1} \sum_{i=1}^{N}\left(\frac{2 i-N-1}{2 N}\right) X_{(i)} & \text { Gini's Mean Difference } \\ D=\frac{2 \sqrt{\pi}}{N(N-1)} \sum_{i=1}^{N}\left(i-\frac{N+1}{2}\right) X_{(i)} & \text { Downton's method } \\ S_{p w}=\frac{\sqrt{\pi}}{N^{2}} \sum_{i=1}^{N}(2 i-N-1) X_{(i)} & \text { Probability Weighted Moments } \\ & \end{array}$

\section{Subscript}

$i$

$j$
For existing estimators

For proposed estimators 


\section{Greek}

$\rho$

$\beta_{1}=\frac{N \sum_{i=1}^{N}\left(X_{i}-\bar{X}\right)^{3}}{(N-1)(N-2) S^{3}}$

$\beta_{2}=\frac{N(N+1) \sum_{i=1}^{N}\left(X_{i}-\bar{X}\right)^{4}}{(N-1)(N-2)(N-3) S^{4}}-\frac{3(N-1)^{2}}{(N-2)(N-3)}$ Coefficient of kurtosis of auxiliary variable

$b=\frac{s_{x y}}{S_{x}^{2}}$
Coefficient of correlation

Coefficient of skewness of auxiliary variable
Regression coefficient of $Y$ on $X$

Based on the above mentioned notations, the mean ratio estimator for estimating the population mean, $\bar{Y}$, of the study variable $Y$ is defined as

$$
\hat{\bar{Y}}_{r}=\frac{\bar{y}}{\bar{x}} \bar{X}
$$

The bias, related constant and the mean squared error (MSE) of the ratio estimator are respectively given by

$$
B\left(\hat{\bar{Y}}_{r}\right)=\frac{(1-f)}{n} \frac{1}{\bar{X}}\left(R S_{x}^{2}-\rho S_{x} S_{y}\right) \quad R=\frac{\bar{Y}}{\bar{X}} \quad \operatorname{MSE}\left(\hat{\bar{Y}}_{r}\right)=\frac{(1-f)}{n}\left(S_{y}^{2}+R^{2} S_{x}^{2}-2 R \rho S_{x} S_{y}\right)
$$

The ratio estimator given in (1) is used for improving the precision of the estimate of the population mean as compared to usual sample mean estimator whenever a positive correlation exists between the study variable and the auxiliary variable. Cochran (1940) suggested a classical ratio type estimator for the estimation of finite population mean using one auxiliary variable under simple random sampling scheme. Murthy (1967) proposed a product type estimator to estimate the population mean or total of study variable by using auxiliary information when coefficient of correlation is negative. Rao (1991) introduced difference type ratio estimator that outperforms conventional ratio and linear regression estimators. Upadhyaya \& Singh (1999) modified ratio type estimators using coefficient of variation and coefficient of kurtosis of the auxiliary variate. Singh \& Tailor (2003) proposed a family of estimators using known values of some parameters by using SRSWOR for estimation of population mean of the study variable. Sisodiaa \& Dwivedi (1981) and Singh et al. (2004) utilized coefficient of variation of the auxiliary variate. Further improvements are achieved by introducing a large number of modified ratio estimators with the use of known coefficient of variation, kurtosis, skewness, median, coefficient of correlation, decile (cf. Subramani and Kumarpandiyan, 2012 a,b,c and $\mathrm{d}$ ).

The organization of the rest of the article is as follows: Section 2 provides a description of the existing estimators. The structure of suggested modified linear regression type ratio estimator and the efficiency comparison of the suggested estimator with the usual ratio estimator and the existing estimators are presented in Section 3. Section 4 consists of an empirical study of proposed estimators. Finally, Section 5 summarizes the findings of the study. 


\section{Existing Ratio Estimators}

Kadilar and Cingi (2004) suggested ratio type estimators for the population mean in the simple random sampling using some known auxiliary information on coefficient of kurtosis and coefficient of variation. They showed that their suggested estimators are more efficient than traditional ratio estimator in the estimation of the population mean.

Kadilar \& Cingi (2004) estimators are given by

$$
\begin{aligned}
& \hat{\bar{Y}}_{1}=\frac{\bar{y}+b(\bar{X}-\bar{x})}{\bar{x}} \bar{X}, \\
& \hat{Y}_{2}=\frac{\bar{y}+b(\bar{X}-\bar{x})}{\left(\bar{x}+C_{x}\right)}\left(\bar{X}+C_{x}\right), \\
& \hat{Y}_{3}=\frac{\bar{y}+b(\bar{X}-\bar{x})}{\left(\bar{x}+\beta_{2}\right)}\left(\bar{X}+\beta_{2}\right), \\
& \hat{Y}_{4}=\frac{\bar{y}+b(\bar{X}-\bar{x})}{\left(\bar{x} \beta_{2}+C_{x}\right)}\left(\bar{X} \beta_{2}+C_{x}\right), \\
& \hat{Y}_{5}=\frac{\bar{y}+b(\bar{X}-\bar{x})}{\left(\bar{x} C_{x}+\beta_{2}\right)}\left(\bar{X} C_{x}+\beta_{2}\right) .
\end{aligned}
$$

The biases, related constants and the MSE for Kadilar and Cingi (2004) estimators are respectively as follows:

$$
\begin{array}{ll}
B\left(\hat{\bar{Y}}_{1}\right)=\frac{(1-f)}{n} \frac{S_{x}^{2}}{\bar{Y}} R_{1}^{2}, R_{1}=\frac{\bar{Y}}{\bar{X}} & \operatorname{MSE}\left(\hat{\bar{Y}}_{1}\right)=\frac{(1-f)}{n}\left(R_{1}^{2} S_{x}^{2}+S_{y}^{2}\left(1-\rho^{2}\right)\right), \\
B\left(\hat{Y}_{2}\right)=\frac{(1-f)}{n} \frac{S_{x}^{2}}{\bar{Y}} R_{2}^{2}, R_{2}=\frac{\bar{Y}}{\left(\bar{X}+C_{x}\right)} & \operatorname{MSE}\left(\hat{\bar{Y}}_{2}\right)=\frac{(1-f)}{n}\left(R_{2}^{2} S_{x}^{2}+S_{y}^{2}\left(1-\rho^{2}\right)\right), \\
B\left(\hat{Y}_{3}\right)=\frac{(1-f)}{n} \frac{S_{x}^{2}}{\bar{Y}} R_{3}^{2}, R_{3}=\frac{\bar{Y}}{\left(\bar{X}+\beta_{2}\right)} & M S E\left(\hat{Y}_{3}\right)=\frac{(1-f)}{n}\left(R_{3}^{2} S_{x}^{2}+S_{y}^{2}\left(1-\rho^{2}\right)\right), \\
B\left(\hat{Y}_{4}\right)=\frac{(1-f)}{n} \frac{S_{x}^{2}}{\bar{Y}} R_{4}^{2}, R_{4}=\frac{\bar{Y} \beta_{2}}{\left(\bar{X} \beta_{2}+C_{x}\right)} & M S E\left(\hat{Y}_{4}\right)=\frac{(1-f)}{n}\left(R_{4}^{2} S_{x}^{2}+S_{y}^{2}\left(1-\rho^{2}\right)\right), \\
B\left(\hat{Y}_{5}\right)=\frac{(1-f)}{n} \frac{S_{x}^{2}}{\bar{Y}} R_{5}^{2}, R_{5}=\frac{\bar{Y} C_{x}}{\left(\bar{X} C_{x}+\beta_{2}\right)} & M S E\left(\hat{Y}_{5}\right)=\frac{(1-f)}{n}\left(R_{5}^{2} S_{x}^{2}+S_{y}^{2}\left(1-\rho^{2}\right)\right) .
\end{array}
$$

Kadilar and Cingi (2006) developed some modified ratio estimators using known value of coefficient of correlation, kurtosis and coefficient of variation as follows:

$$
\begin{aligned}
& \hat{\bar{Y}}_{6}=\frac{\bar{y}+b(\bar{X}-\bar{x})}{\bar{x}}(\bar{X}+\rho), \\
& \hat{Y}_{7}=\frac{\bar{y}+b(\bar{X}-\bar{x})}{\left(\bar{x} c_{x}+\rho\right)}\left(\bar{X} C_{x}+\rho\right), \\
& \hat{\bar{Y}}_{8}=\frac{\bar{y}+b(\bar{X}-\bar{x})}{\left(\bar{x} \rho+C_{x}\right)}\left(\bar{X} \rho+C_{x}\right), \\
& \hat{\bar{Y}}_{9}=\frac{\bar{y}+b(\bar{X}-\bar{x})}{\left(\bar{x} \beta_{2}+\rho\right)}\left(\bar{X} \beta_{2}+\rho\right), \\
& \hat{\bar{Y}}_{10}=\frac{\bar{y}+b(\bar{X}-\bar{x})}{\left(\bar{x} \rho+\beta_{2}\right)}\left(\bar{X} \rho+\beta_{2}\right) .
\end{aligned}
$$


The biases, related constants and the MSE for Kadilar and Cingi (2006) estimators are respectively given by

$$
\begin{array}{lll}
B\left(\hat{\bar{Y}}_{6}\right)=\frac{(1-f)}{n} \frac{S_{x}^{2}}{\bar{Y}} R_{6}^{2}, & R_{6}=\frac{\bar{Y}}{\bar{X}+\rho} & M S E\left(\hat{\bar{Y}}_{6}\right)=\frac{(1-f)}{n}\left(R_{6}^{2} S_{x}^{2}+S_{y}^{2}\left(1-\rho^{2}\right)\right), \\
B\left(\hat{\bar{Y}}_{7}\right)=\frac{(1-f)}{n} \frac{S_{x}^{2}}{\bar{Y}} R_{7}^{2}, & R_{7}=\frac{\bar{Y} C_{x}}{\left(\bar{X} C_{x}+\rho\right)} & M S E\left(\hat{\bar{Y}}_{7}\right)=\frac{(1-f)}{n}\left(R_{7}^{2} S_{x}^{2}+S_{y}^{2}\left(1-\rho^{2}\right)\right), \\
B\left(\hat{\bar{Y}}_{8}\right)=\frac{(1-f)}{n} \frac{S_{x}^{2}}{\bar{Y}} R_{8}^{2}, & R_{8}=\frac{\bar{Y} \rho}{\left(\bar{X} \rho+C_{x}\right)} & M S E\left(\hat{\bar{Y}}_{8}\right)=\frac{(1-f)}{n}\left(R_{8}^{2} S_{x}^{2}+S_{y}^{2}\left(1-\rho^{2}\right)\right), \\
B\left(\hat{\bar{Y}}_{9}\right)=\frac{(1-f)}{n} \frac{S_{x}^{2}}{\bar{Y}} R_{9}^{2}, & R_{9}=\frac{\bar{Y} \beta_{2}}{\left(\bar{X} \beta_{2}+\rho\right)} & M S E\left(\hat{\bar{Y}}_{9}\right)=\frac{(1-f)}{n}\left(R_{9}^{2} S_{x}^{2}+S_{y}^{2}\left(1-\rho^{2}\right)\right), \\
B\left(\hat{\bar{Y}}_{10}\right)=\frac{(1-f)}{n} \frac{S_{x}^{2}}{\bar{Y}} R_{10}^{2}, & R_{10}=\frac{\bar{Y} \rho}{\left(\bar{X} \rho+\beta_{2}\right)} & M S E\left(\hat{\bar{Y}}_{10}\right)=\frac{(1-f)}{n}\left(R_{10}^{2} S_{x}^{2}+S_{y}^{2}\left(1-\rho^{2}\right)\right) .
\end{array}
$$

Yan and Tian (2010) proposed some modified ratio estimators using coefficient of skewness and kurtosis as follows:

$$
\begin{aligned}
& \hat{\bar{Y}}_{11}=\frac{\bar{y}+b(\bar{X}-\bar{x})}{\left(\bar{x}+\beta_{1}\right)}\left(\bar{X}+\beta_{1}\right), \\
& \hat{\bar{Y}}_{12}=\frac{\bar{y}+b(\bar{X}-\bar{x})}{\left(\bar{x} \beta_{1}+\beta_{2}\right)}\left(\bar{X} \beta_{1}+\beta_{2}\right) .
\end{aligned}
$$

The biases, related constants and the MSE for Yan and Tian (2010) estimators are respectively given by

$$
\begin{array}{lll}
B\left(\hat{\bar{Y}}_{11}\right)=\frac{(1-f)}{n} \frac{S_{x}^{2}}{\bar{Y}} R_{11}^{2}, & R_{11}=\frac{\bar{Y}}{\left(\bar{X}+\beta_{1}\right)} & \operatorname{MSE}\left(\hat{\bar{Y}}_{11}\right)=\frac{(1-f)}{n}\left(R_{11}^{2} S_{x}^{2}+S_{y}^{2}\left(1-\rho^{2}\right)\right), \\
B\left(\hat{\bar{Y}}_{12}\right)=\frac{(1-f)}{n} \frac{S_{x}^{2}}{\bar{Y}} R_{12}^{2}, & R_{12}=\frac{\bar{Y} \beta_{1}}{\left(\bar{X} \beta_{1}+\beta_{2}\right)} & M S E\left(\hat{\bar{Y}}_{12}\right)=\frac{(1-f)}{n}\left(R_{12}^{2} S_{x}^{2}+S_{y}^{2}\left(1-\rho^{2}\right)\right) .
\end{array}
$$

Yan and Tian (2010) showed that the use of coefficient of skewness and coefficient of kurtosis, respectively, provides better estimates for the population mean in comparison to the usual ratio estimator and numerous existing estimators.

Subramani and Kumarapandiyan (2012a, 2012b, 2012c) introduced the following estimators with the use of population median, skewness, kurtosis and coefficient of variation of auxiliary information in the simple random sampling for the estimation of the population mean.

$$
\begin{aligned}
& \hat{\bar{Y}}_{13}=\frac{\bar{y}+b(\bar{X}-\bar{x})}{\left(\bar{x}+M_{d}\right)}\left(\bar{X}+M_{d}\right), \\
& \hat{\bar{Y}}_{14}=\frac{\bar{y}+b(\bar{X}-\bar{x})}{\left(\bar{x} C_{x}+M_{d}\right)}\left(\bar{X} C_{x}+M_{d}\right), \\
& \hat{\bar{Y}}_{15}=\frac{\bar{y}+b(\bar{X}-\bar{x})}{\left(\bar{x} \beta_{1}+M_{d}\right)}\left(\bar{X} \beta_{1}+M_{d}\right), \\
& \hat{\bar{Y}}_{16}=\frac{\bar{y}+b(\bar{x}-\bar{x})}{\left(\bar{x} \beta_{2}+M_{d}\right)}\left(\bar{X} \beta_{2}+M_{d}\right) .
\end{aligned}
$$


The biases, related constant and the MSE for Subramani and Kumarapandiyan (2012a, 2012 b, 2012c) estimators are respectively given by

$$
\begin{array}{lll}
B\left(\hat{\bar{Y}}_{13}\right)=\frac{(1-f)}{n} \frac{S_{x}^{2}}{\bar{Y}} R_{13}^{2}, & R_{13}=\frac{\bar{Y}}{\left(\bar{X}+M_{d}\right)} & M S E\left(\hat{\bar{Y}}_{13}\right)=\frac{(1-f)}{n}\left(R_{13}^{2} S_{x}^{2}+S_{y}^{2}\left(1-\rho^{2}\right)\right), \\
B\left(\hat{\bar{Y}}_{14}\right)=\frac{(1-f)}{n} \frac{S_{x}^{2}}{\bar{Y}} R_{14}^{2}, & R_{14}=\frac{\bar{Y} C_{x}}{\left(\bar{X} C_{x}+M_{d}\right)} & M S E\left(\hat{\bar{Y}}_{14}\right)=\frac{(1-f)}{n}\left(R_{14}^{2} S_{x}^{2}+S_{y}^{2}\left(1-\rho^{2}\right)\right), \\
B\left(\hat{\bar{Y}}_{15}\right)=\frac{(1-f)}{n} \frac{S_{x}^{2}}{\bar{Y}} R_{15}^{2}, & R_{15}=\frac{\bar{Y} \beta_{1}}{\left(\bar{X} \beta_{1}+M_{d}\right)} & M S E\left(\hat{\bar{Y}}_{15}\right)=\frac{(1-f)}{n}\left(R_{15}^{2} S_{x}^{2}+S_{y}^{2}\left(1-\rho^{2}\right)\right), \\
B\left(\hat{\bar{Y}}_{14}\right)=\frac{(1-f)}{n} \frac{S_{x}^{2}}{\bar{Y}} R_{14}^{2}, & R_{16}=\frac{\bar{Y} \beta_{2}}{\left(\bar{X} \beta_{2}+M_{d}\right)} & M S E\left(\hat{\bar{Y}}_{16}\right)=\frac{(1-f)}{n}\left(R_{16}^{2} S_{x}^{2}+S_{y}^{2}\left(1-\rho^{2}\right)\right) .
\end{array}
$$

Jeelani et al. (2013) suggested an estimator with the use of coefficient of skweness and quartile deviation of the auxiliary information in the simple random sampling for the estimation of the population mean as follows:

$\hat{\bar{Y}}_{17}=\frac{\bar{y}+b(\bar{X}-\bar{x})}{\left(\bar{x} \beta_{1}+Q D\right)}\left(\bar{X} \beta_{1}+Q D\right)$.

The bias, related constant and the MSE for Jeelani et al. (2013) estimator is given by

$$
B\left(\hat{\bar{Y}}_{17}\right)=\frac{(1-f)}{n} \frac{S_{x}^{2}}{\bar{Y}} R_{17}^{2}, \quad R_{17}=\frac{\bar{Y} \beta_{1}}{\left(\bar{X} \beta_{1}+Q D\right)} \quad \operatorname{MSE}\left(\hat{\bar{Y}}_{17}\right)=\frac{(1-f)}{n}\left(R_{17}^{2} S_{x}^{2}+S_{y}^{2}\left(1-\rho^{2}\right)\right) .
$$

\section{Proposed Modified Ratio Estimators}

Motivated by the mentioned estimators in Section 2, we propose some new modified ratio type estimators. It is relevant to note that the measures like range, variance, standard deviation and mean deviation are affected by extreme values in the population, whereas the Gini's mean difference, Downton's method and probability weighted moments measures are robust and are more effective in the presence of outliers in the population. The proposed estimators using the linear combination of population coefficient of variation, population coefficient of correlation, Gini's mean difference estimator, Downton's method and probability weighted moments can be formulated as follows:

$$
\begin{aligned}
& \hat{\bar{Y}}_{p 1}=\frac{\bar{y}+b(\bar{X}-\bar{x})}{(\bar{x}+G)}(\bar{X}+G), \\
& \hat{\bar{Y}}_{p 2}=\frac{\bar{y}+b(\bar{X}-\bar{x})}{(\bar{x} \rho+G)}(\bar{X} \rho+G), \\
& \hat{\bar{Y}}_{p 3}=\frac{\bar{y}+b(\bar{X}-\bar{x})}{\left(\bar{x} C_{x}+G\right)}\left(\bar{X} C_{x}+G\right), \\
& \hat{\bar{Y}}_{p 4}=\frac{\bar{y}+b(\bar{X}-\bar{x})}{(\bar{x}+D)}(\bar{X}+D), \\
& \hat{\bar{Y}}_{p 5}=\frac{\bar{y}+b(\bar{X}-\bar{x})}{(\bar{x} \rho+D)}(\bar{X} \rho+D), \\
& \hat{\bar{Y}}_{p 6}=\frac{\bar{y}+b(\bar{X}-\bar{x})}{\left(\bar{x} C_{x}+D\right)}\left(\bar{X} C_{x}+D\right), \\
& \hat{\bar{Y}}_{p 7}=\frac{\bar{y}+b(\bar{X}-\bar{x})}{\left(\bar{x}+S_{p w}\right)}\left(\bar{X}+S_{p w}\right),
\end{aligned}
$$




$$
\begin{aligned}
& \hat{\bar{Y}}_{p 8}=\frac{\bar{y}+b(\bar{X}-\bar{x})}{\left(\bar{x} \rho+S_{p w}\right)}\left(\bar{X} \rho+S_{p w}\right), \\
& \hat{\bar{Y}}_{p 9}=\frac{\bar{y}+b(\bar{X}-\bar{x})}{\left(\bar{x} C_{x}+S_{p w}\right)}\left(\bar{X} C_{x}+S_{p w}\right) .
\end{aligned}
$$

The biases, related constants and the MSE for suggested estimators can be obtained as follows:

$$
\begin{array}{lll}
B\left(\hat{\bar{Y}}_{p 1}\right)=\frac{(1-f)}{n} \frac{S_{x}^{2}}{\bar{Y}} R_{p 1}^{2}, & R_{p 1}=\frac{\bar{Y}}{(\bar{X}+G)} & M S E\left(\hat{\bar{Y}}_{p 1}\right)=\frac{(1-f)}{n}\left(R_{p 1}^{2} S_{x}^{2}+S_{y}^{2}\left(1-\rho^{2}\right)\right), \\
B\left(\hat{\bar{Y}}_{p 2}\right)=\frac{(1-f)}{n} \frac{S_{x}^{2}}{\bar{Y}} R_{p 2}^{2}, & R_{p 2}=\frac{\bar{Y} \rho}{(\bar{X} \rho+G)} & M S E\left(\hat{\bar{Y}}_{p 2}\right)=\frac{(1-f)}{n}\left(R_{p 2}^{2} S_{x}^{2}+S_{y}^{2}\left(1-\rho^{2}\right)\right), \\
B\left(\hat{\bar{Y}}_{p 3}\right)=\frac{(1-f)}{n} \frac{S_{x}^{2}}{\bar{Y}} R_{p 3}^{2}, & R_{p 3}=\frac{\bar{Y} C_{x}}{\left(\bar{X} C_{x}+G\right)} & M S E\left(\hat{\bar{Y}}_{p 3}\right)=\frac{(1-f)}{n}\left(R_{p 3}^{2} S_{x}^{2}+S_{y}^{2}\left(1-\rho^{2}\right)\right), \\
B\left(\hat{\bar{Y}}_{p 4}\right)=\frac{(1-f)}{n} \frac{S_{x}^{2}}{\bar{Y}} R_{p 4}^{2}, & R_{p 4}=\frac{\bar{Y}}{(\bar{X}+D)} & M S E\left(\hat{\bar{Y}}_{p 4}\right)=\frac{(1-f)}{n}\left(R_{p 4}^{2} S_{x}^{2}+S_{y}^{2}\left(1-\rho^{2}\right)\right), \\
B\left(\hat{\bar{Y}}_{p 5}\right)=\frac{(1-f)}{n} \frac{S_{x}^{2}}{\bar{Y}} R_{p 5}^{2}, & R_{p 5}=\frac{\bar{Y} \rho}{(\bar{X} \rho+D)} & M S E\left(\hat{\bar{Y}}_{p 5}\right)=\frac{(1-f)}{n}\left(R_{p 5}^{2} S_{x}^{2}+S_{y}^{2}\left(1-\rho^{2}\right)\right), \\
B\left(\hat{\bar{Y}}_{p 6}\right)=\frac{(1-f)}{n} \frac{S_{x}^{2}}{\bar{Y}} R_{p 6}^{2}, & R_{p 6}=\frac{\bar{Y} C_{x}}{\left(\bar{X} C_{x}+D\right)} & M S E\left(\hat{\bar{Y}}_{p 6}\right)=\frac{(1-f)}{n}\left(R_{p 6}^{2} S_{x}^{2}+S_{y}^{2}\left(1-\rho^{2}\right)\right), \\
B\left(\hat{\bar{Y}}_{p 7}\right)=\frac{(1-f)}{n} \frac{S_{x}^{2}}{\bar{Y}} R_{p 7}^{2}, & R_{p 7}=\frac{\bar{Y}}{\left(\bar{X}+S_{p w}\right)} & M S E\left(\hat{\bar{Y}}_{p 7}\right)=\frac{(1-f)}{n}\left(R_{p 7}^{2} S_{x}^{2}+S_{y}^{2}\left(1-\rho^{2}\right)\right), \\
B\left(\hat{\bar{Y}}_{p 8}\right)=\frac{(1-f)}{n} \frac{S_{x}^{2}}{\bar{Y}} R_{p 8}^{2}, & R_{p 8}=\frac{\bar{Y} \rho}{\left(\bar{X} \rho+S_{p w}\right)} & M S E\left(\hat{\bar{Y}}_{p 8}\right)=\frac{(1-f)}{n}\left(R_{p 8}^{2} S_{x}^{2}+S_{y}^{2}\left(1-\rho^{2}\right)\right), \\
B\left(\hat{\bar{Y}}_{p 9}\right)=\frac{(1-f)}{n} \frac{S_{x}^{2}}{\bar{Y}} R_{p 9}^{2}, & R_{p 9}=\frac{\bar{Y} C_{x}}{\left(\bar{X} C_{x}+S_{p w}\right)} & M S E\left(\hat{\bar{Y}}_{p 9}\right)=\frac{(1-f)}{n}\left(R_{p 9}^{2} S_{x}^{2}+S_{y}^{2}\left(1-\rho^{2}\right)\right) .
\end{array}
$$

\section{Efficiency Comparisons}

In this section, the efficiency conditions for the proposed ratio estimators have been

derived algebraically according to usual ratio estimator and existing ratio estimators in literature.

\subsection{Comparison with usual ratio estimator}

The proposed ratio estimators are more efficient than that of the usual ratio estimator if

$$
\begin{aligned}
& \operatorname{MSE}\left(\hat{\bar{Y}}_{p j}\right) \leq \operatorname{MSE}\left(\hat{\bar{Y}}_{r}\right) \\
& \frac{(1-f)}{n}\left(R_{p j}^{2} S_{x}^{2}+S_{y}^{2}\left(1-\rho^{2}\right)\right) \leq \frac{(1-f)}{n}\left(S_{y}^{2}+R^{2} S_{x}^{2}-2 R \rho S_{x} S_{y}\right) \\
& R_{p j}^{2} S_{x}^{2}-\rho^{2} S_{y}^{2}-R^{2} S_{x}^{2}+2 R \rho S_{x} S_{y} \leq 0 \\
& \left(\rho S_{y}-R S_{x}\right)^{2}-R_{p j}^{2} S_{x}^{2} \geq 0 \\
& \left(\rho S_{y}-R S_{x}+R_{p j} S_{x}\right)\left(\rho S_{y}-R S_{x}-R_{p j} S_{x}\right) \geq 0
\end{aligned}
$$


Condition I: $\left(\rho S_{y}-R S_{x}+R_{p j} S_{x}\right) \leq 0$ and $\left(\rho S_{y}-R S_{x}-R_{p j} S_{x}\right) \leq 0$

After solving the condition I, we get

$$
\left(\frac{\rho S_{y}-R S_{x}}{S_{x}}\right) \leq R_{p j} \leq\left(\frac{R S_{x}-\rho S_{y}}{S_{x}}\right)
$$

Condition II: $\left(\rho S_{y}-R S_{x}+R_{p j} S_{x}\right) \geq 0$ and $\left(\rho S_{y}-R S_{x}-R_{p j} S_{x}\right) \geq 0$

After simplifying the Condition II, we get

$$
\left(\frac{R S_{x}-\rho S_{y}}{S_{x}}\right) \leq R_{p j} \leq\left(\frac{\rho S_{y}-R S_{x}}{S_{x}}\right)
$$

Hence,

$$
\begin{aligned}
& \operatorname{MSE}\left(\hat{\bar{Y}}_{p j}\right) \leq \operatorname{MSE}\left(\hat{\bar{Y}}_{r}\right), \\
& \left(\frac{\rho S_{y}-R S_{x}}{S_{x}}\right) \leq R_{p j} \leq\left(\frac{R S_{x}-\rho S_{y}}{S_{x}}\right),
\end{aligned}
$$

or

$$
\left(\frac{R S_{x}-\rho S_{y}}{S_{x}}\right) \leq R_{p j} \leq\left(\frac{\rho S_{y}-R S_{x}}{S_{x}}\right) . \quad \text { where } j=1,2, \ldots, 9
$$

\subsection{Comparisons with existing ratio estimators}

From the expressions of the MSE of the proposed estimators and the existing estimators, we have derived the conditions for which the proposed estimators are more efficient than the existing modified ratio estimators as follows:

$$
\begin{aligned}
& \operatorname{MSE}\left(\hat{\bar{Y}}_{p j}\right) \leq \operatorname{MSE}\left(\hat{\bar{Y}}_{i}\right) \\
& \frac{(1-f)}{n}\left(R_{p j}^{2} S_{x}^{2}+S_{y}^{2}\left(1-\rho^{2}\right)\right) \leq \frac{(1-f)}{n}\left(R_{i}^{2} S_{x}^{2}+S_{y}^{2}\left(1-\rho^{2}\right)\right) \\
& R_{p j}^{2} S_{x}^{2} \leq R_{i}^{2} S_{x}^{2} \\
& R_{p j} \leq R_{i}
\end{aligned}
$$

where $j=1,2, \ldots, 9$ and $i=1,2, \ldots, 17$.

\section{Empirical Study}

The performances of the suggested ratio estimators are evaluated and compared with the usual ratio estimator and the mentioned ratio estimators in Section 2 by using 4 natural populations. The Population 1 is taken from page 177 of Singh and Chaudhary (1986), Populations 2 and 3 are taken from page 228 of Murthy (1967), and the Population 4 is taken from Kadilar and Cingi (2004). 
The statistics of 4 populations are given as follows:

Population 1: Singh and Chaudhary (1986)

$\begin{array}{llll}N=34 & n=20 & \bar{Y}=856.4117 & \bar{X}=199.4412 \\ \rho=0.4453 & S_{y}=733.1407 & C_{y}=0.8561 & S_{x}=150.2150 \\ C_{x}=0.7531 & \beta_{2}=1.0445 & \beta_{1}=1.1823 & M_{d}=142.50 \\ Q D=80.25 & G=162.996 & D=144.481 & S_{p w}=142.990\end{array}$

Population 2: Murthy (1967)

$\begin{array}{llll}N=80 & n=20 & \bar{Y}=5182.637 & \bar{X}=285.125 \\ \rho=0.915 & S_{y}=1835.659 & C_{y}=0.3540 & S_{x}=279.429 \\ C_{x}=0.948 & \beta_{2}=1.301 & \beta_{1}=0.698 & M_{d}=148.00 \\ Q D=179.375 & G=279.711 & D=247.938 & S_{p w}=244.838\end{array}$

Population 3: Murthy (1967)

$\begin{array}{llll}N=80 & n=20 & \bar{Y}=5182.637 & \bar{X}=1126.463 \\ \rho=0.941 & S_{y}=1835.659 & C_{y}=0.3540 & S_{x}=845.610 \\ C_{x}=0.751 & \beta_{2}=-0.063 & \beta_{1}=1.050 & M_{d}=757.500 \\ Q D=588.125 & G=904.081 & D=801.381 & S_{p w}=791.364\end{array}$

Population 4: Kadilar and Cingi (2004)

$\begin{array}{llll}N=106 & n=40 & \bar{Y}=2212.59 & \bar{X}=27421.70 \\ \rho=0.860 & S_{y}=11551.53 & C_{y}=5.22 & S_{x}=57460.61 \\ C_{x}=2.10 & \beta_{2}=34.572 & \beta_{1}=2.122 & M_{d}=7297.50 \\ Q D=12156.25 & G=40201.69 & D=35634.990 & S_{p w}=35298.810\end{array}$

The values of the related constants and the biases of the existing and proposed modified ratio estimators are given in Table 1, whereas the values of the MSE of the existing and proposed estimators are given in Table 2. 
Muhammad Abid, Nasir Abbas, Rehan Ahmad Khan Sherwani, Hafiz Zafar Nazir

Table 1: The related constants and biases of the existing and the proposed ratio estimators

\begin{tabular}{|c|c|c|c|c|c|c|c|c|}
\hline \multirow[b]{2}{*}{ Estimator } & \multicolumn{4}{|c|}{ Constant } & \multicolumn{4}{|c|}{ Bias } \\
\hline & $\begin{array}{c}\text { Population } \\
1\end{array}$ & $\begin{array}{c}\text { Population } \\
2\end{array}$ & $\begin{array}{c}\text { Population } \\
\mathbf{3}\end{array}$ & $\begin{array}{c}\text { Population } \\
4\end{array}$ & $\begin{array}{c}\text { Population } \\
1\end{array}$ & $\begin{array}{c}\text { Population } \\
2\end{array}$ & $\begin{array}{c}\text { Population } \\
\mathbf{3}\end{array}$ & $\begin{array}{c}\text { Population } \\
4\end{array}$ \\
\hline$\widehat{\bar{Y}}_{r}$ & 4.294 & 18.177 & 4.601 & 0.0807 & 4.94 & 115.09 & 60.88 & 171.32 \\
\hline$\widehat{\bar{Y}}_{1}$ & 4.294 & 18.177 & 4.601 & 0.0807 & 10.00 & 174.83 & 109.52 & 151.20 \\
\hline$\widehat{\bar{Y}}_{2}$ & 4.294 & 18.177 & 4.601 & 0.0807 & 9.93 & 173.67 & 109.37 & 151.18 \\
\hline$\widehat{\bar{Y}}_{3}$ & 4.278 & 18.116 & 4.598 & 0.0806 & 9.89 & 173.98 & 109.53 & 150.82 \\
\hline$\widehat{\bar{Y}}_{4}$ & 4.272 & 18.132 & 4.601 & 0.0807 & 9.93 & 173.18 & 111.86 & 151.20 \\
\hline$\widehat{\widehat{Y}}_{5}$ & 4.279 & 18.090 & 4.650 & 0.0806 & 9.87 & 173.93 & 109.53 & 151.02 \\
\hline$\widehat{\bar{Y}}_{6}$ & 4.264 & 18.130 & 4.601 & 0.0807 & 9.96 & 173.71 & 109.34 & 151.19 \\
\hline$\widehat{\bar{Y}}_{7}$ & 4.285 & 18.119 & 4.597 & 0.0807 & 9.94 & 173.65 & 109.27 & 151.20 \\
\hline$\widehat{\bar{Y}}_{8}$ & 4.281 & 18.115 & 4.596 & 0.0807 & 9.83 & 173.57 & 109.36 & 151.17 \\
\hline$\widehat{\bar{Y}}_{9}$ & 4.258 & 18.111 & 4.598 & 0.8067 & 9.96 & 173.23 & 112.46 & 151.20 \\
\hline$\widehat{\bar{Y}}_{10}$ & 4.285 & 18.094 & 4.662 & 0.0806 & 9.77 & 173.90 & 109.53 & 150.76 \\
\hline$\widehat{\widehat{Y}}_{11}$ & 4.244 & 18.128 & 4.601 & 0.0807 & 9.89 & 173.24 & 109.31 & 151.14 \\
\hline$\widehat{\bar{Y}}_{12}$ & 4.269 & 18.094 & 4.597 & 0.0807 & 9.91 & 174.17 & 109.53 & 151.13 \\
\hline$\widehat{\widehat{Y}}_{13}$ & 4.275 & 18.143 & 4.601 & 0.0637 & 3.40 & 75.76 & 39.15 & 94.32 \\
\hline$\widehat{\bar{Y}}_{14}$ & 2.504 & 11.966 & 2.751 & 0.0715 & 2.63 & 73.02 & 30.47 & 119.04 \\
\hline$\widehat{\bar{Y}}_{15}$ & 2.204 & 11.747 & 2.427 & 0.0767 & 3.89 & 89.31 & 40.69 & 136.64 \\
\hline$\widehat{\bar{Y}}_{16}$ & 2.676 & 12.991 & 2.804 & 0.0801 & 3.53 & 57.48 & 1.186 & 148.10 \\
\hline$\widehat{\bar{Y}}_{17}$ & 2.550 & 10.422 & 0.478 & 0.0742 & 5.26 & 79.42 & 48.85 & 128.08 \\
\hline$\widehat{\widehat{Y}}_{p 1}$ & 2.363 & 9.175 & 2.552 & 0.0327 & 3.03 & 44.55 & 33.71 & 24.87 \\
\hline$\widehat{\widehat{Y}}_{p 2}$ & 2.059 & 8.935 & 2.224 & 0.0475 & 2.30 & 42.25 & 25.58 & 52.34 \\
\hline$\widehat{\bar{Y}}_{p 3}$ & 1.515 & 8.772 & 2.483 & 0.0297 & 1.24 & 40.72 & 31.91 & 20.59 \\
\hline$\widehat{\widehat{Y}}_{p 4}$ & 1.635 & 9.320 & 2.620 & 0.0320 & 1.45 & 45.96 & 35.53 & 23.85 \\
\hline$\widehat{\bar{Y}}_{p 5}$ & 2.189 & 9.483 & 2.362 & 0.0498 & 2.60 & 47.58 & 28.87 & 57.60 \\
\hline$\widehat{\widehat{Y}}_{p 6}$ & 2.490 & 9.722 & 2.688 & 0.0351 & 3.36 & 50.02 & 37.39 & 28.59 \\
\hline$\widehat{\widehat{Y}}_{p 7}$ & 1.645 & 9.377 & 2.635 & 0.0322 & 1.47 & 46.53 & 35.91 & 24.12 \\
\hline$\widehat{\bar{Y}}_{p 8}$ & 2.200 & 9.540 & 2.377 & 0.0500 & 2.63 & 48.16 & 29.22 & 58.02 \\
\hline$\widehat{\widehat{Y}}_{p 9}$ & 2.501 & 9.779 & 2.702 & 0.0353 & 3.39 & 50.61 & 37.78 & 28.90 \\
\hline
\end{tabular}


Table 2: The MSE values of the existing and the proposed ratio estimators

\begin{tabular}{|c|c|c|c|c|}
\hline \multirow{2}{*}{ Estimator } & \multicolumn{4}{|c|}{ Mean square Error } \\
\hline & Population 1 & Population 2 & Population 3 & Population 4 \\
\hline$\widehat{\bar{Y}}_{r}$ & 10960.76 & 413243.60 & 189775.10 & 984589.70 \\
\hline$\widehat{\widehat{Y}}_{1}$ & 17437.65 & 926660.70 & 581994.20 & 889617.50 \\
\hline$\widehat{\bar{Y}}_{2}$ & 17373.31 & 920662.50 & 581238.50 & 889566.40 \\
\hline$\widehat{\bar{Y}}_{3}$ & 17348.62 & 922242.50 & 582058.10 & 888775.70 \\
\hline$\widehat{\widehat{Y}}_{4}$ & 17376.04 & 918082.10 & 594119.80 & 889616.00 \\
\hline$\widehat{\bar{Y}}_{5}$ & 17319.75 & 922003.40 & 582079.30 & 889215.30 \\
\hline$\widehat{\bar{Y}}_{6}$ & 17399.52 & 920873.20 & 581046.80 & 889596.60 \\
\hline$\widehat{\widehat{Y}}_{7}$ & 17387.08 & 920560.30 & 580732.70 & 889607.50 \\
\hline$\widehat{\bar{Y}}_{8}$ & 17294.19 & 920108.20 & 581191.40 & 889557.80 \\
\hline$\widehat{\bar{Y}}_{9}$ & 17401.14 & 918382.80 & 597260.90 & 889616.90 \\
\hline$\widehat{\widehat{Y}}_{10}$ & 17239.66 & 921833.60 & 582062.10 & 888634.40 \\
\hline$\widehat{\widehat{Y}}_{11}$ & 17336.98 & 918450.90 & 580937.60 & 889492.50 \\
\hline$\widehat{\bar{Y}}_{12}$ & 17362.26 & 923260.70 & 582055.10 & 889452.90 \\
\hline$\widehat{\bar{Y}}_{13}$ & 11785.70 & 413230.80 & 217319.80 & 763783.60 \\
\hline$\widehat{\bar{Y}}_{14}$ & 11127.47 & 399044.90 & 172323.80 & 818477.40 \\
\hline$\widehat{\bar{Y}}_{15}$ & 12199.76 & 483450.40 & 225319.50 & 857402.20 \\
\hline$\widehat{\widehat{Y}}_{16}$ & 11892.07 & 318486.70 & 20545.47 & 884526.80 \\
\hline$\widehat{\widehat{Y}}_{17}$ & 13376.04 & 432164.60 & 267595.20 & 838466.80 \\
\hline$\widehat{\bar{Y}}_{p 1}$ & 11465.43 & 251457.80 & 189080.30 & 610126.10 \\
\hline$\widehat{\bar{Y}}_{p 2}$ & 10841.88 & 239515.60 & 146971.80 & 670914.00 \\
\hline$\widehat{\bar{Y}}_{p 3}$ & 9937.20 & 231591.30 & 179770.50 & 600579.70 \\
\hline$\widehat{\widehat{Y}}_{p 4}$ & 10113.06 & 258768.30 & 198518.70 & 607875.10 \\
\hline$\widehat{\bar{Y}}_{p 5}$ & 11097.24 & 267178.40 & 164020.70 & 682552.70 \\
\hline$\widehat{\widehat{Y}}_{p 6}$ & 11752.23 & 279802.00 & 208187.20 & 618381.50 \\
\hline$\widehat{\bar{Y}}_{p 7}$ & 10129.01 & 261697.40 & 200516.20 & 608480.30 \\
\hline$\widehat{\bar{Y}}_{p 8}$ & 11119.84 & 270154.60 & 165857.40 & 683478.00 \\
\hline$\widehat{\bar{Y}}_{p 9}$ & 11777.26 & 282843.60 & 210216.90 & 619061.50 \\
\hline
\end{tabular}

It can be observed that the related constants and the biases of the suggested modified ratio estimators are smaller than the usual ratio estimator and the existing ratio estimators in literature. From Table 2, it is obvious that the proposed estimators perform better than the usual ratio estimator and the existing modified ratio estimators in terms of MSE, which indicates that the proposed estimators are more efficient.

To get more insight into the proposals of the study, we have also evaluated the percentage relative efficiencies (PRE) of the proposed estimators (p) with respect to the existing estimators (e), which is computed by the formula given below

$$
\operatorname{PRE}(e, p)=\frac{\operatorname{MSE}(e)}{\operatorname{MSE}(p)} \times 100
$$

and the values of PRE are given in Tables $3-6$. 
Table 3: PRE of the proposed estimators and the existing estimators for population 1

\begin{tabular}{|c|c|c|c|c|c|c|c|c|c|}
\hline \multirow{2}{*}{$\begin{array}{c}\text { Existing } \\
\text { Estimators }\end{array}$} & \multicolumn{9}{|c|}{ Proposed Estimators } \\
\hline & $\widehat{\bar{Y}}_{p 1}$ & $\widehat{\bar{Y}}_{p 2}$ & $\widehat{\bar{Y}}_{p 3}$ & $\widehat{\widehat{Y}}_{p 4}$ & $\widehat{\bar{Y}}_{p 5}$ & $\widehat{\widehat{Y}}_{p 6}$ & $\widehat{\widehat{Y}}_{p 7}$ & $\widehat{\widehat{Y}}_{p 8}$ & $\widehat{\overline{\boldsymbol{Y}}}_{p 9}$ \\
\hline$\widehat{\widehat{Y}}_{r}$ & 95.6 & 101.1 & 110.3 & 108.4 & 98.8 & 93.3 & 108.2 & 98.6 & 93.1 \\
\hline$\widehat{\widehat{Y}}_{1}$ & 152.1 & 160.8 & 175.5 & 172.4 & 157.1 & 148.4 & 172.2 & 156.8 & 148.1 \\
\hline$\widehat{\widehat{Y}}_{2}$ & 151.5 & 160.2 & 174.8 & 171.8 & 156.6 & 147.8 & 171.5 & 156.2 & 147.5 \\
\hline$\widehat{\widehat{Y}}_{3}$ & 151.3 & 160.0 & 174.6 & 171.5 & 156.3 & 147.6 & 171.3 & 156.0 & 147.3 \\
\hline$\widehat{\widehat{Y}}_{4}$ & 151.6 & 160.3 & 174.9 & 171.8 & 156.6 & 147.9 & 171.5 & 156.3 & 147.5 \\
\hline$\widehat{\widehat{Y}}_{5}$ & 151.1 & 159.7 & 174.3 & 171.3 & 156.1 & 147.4 & 171.0 & 155.8 & 147.1 \\
\hline$\widehat{\widehat{Y}}_{6}$ & 151.8 & 160.5 & 175.1 & 172.1 & 156.8 & 148.1 & 171.8 & 156.5 & 147.7 \\
\hline$\widehat{\widehat{Y}}_{7}$ & 151.6 & 160.4 & 175.0 & 171.9 & 156.7 & 147.9 & 171.7 & 156.4 & 147.6 \\
\hline$\widehat{\widehat{Y}}_{8}$ & 150.8 & 159.5 & 174.0 & 171.0 & 155.8 & 147.2 & 170.7 & 155.5 & 146.8 \\
\hline$\widehat{\bar{Y}}_{9}$ & 151.8 & 160.5 & 175.1 & 172.1 & 156.8 & 148.1 & 171.8 & 156.5 & 147.8 \\
\hline$\widehat{\bar{Y}}_{10}$ & 150.4 & 159.0 & 173.5 & 170.5 & 155.4 & 146.7 & 170.2 & 155.0 & 146.4 \\
\hline$\widehat{\bar{Y}}_{11}$ & 151.2 & 159.9 & 174.5 & 171.4 & 156.2 & 147.5 & 171.2 & 155.9 & 147.2 \\
\hline$\widehat{\widehat{Y}}_{12}$ & 151.4 & 160.1 & 174.7 & 171.7 & 156.5 & 147.7 & 171.4 & 156.1 & 147.4 \\
\hline$\widehat{\bar{Y}}_{13}$ & 102.8 & 108.7 & 118.6 & 116.5 & 106.2 & 100.3 & 116.4 & 106.0 & 100.1 \\
\hline$\widehat{\widehat{Y}}_{14}$ & 97.1 & 102.6 & 112.0 & 110.0 & 100.3 & 94.7 & 109.9 & 100.1 & 94.5 \\
\hline$\widehat{\bar{Y}}_{15}$ & 106.4 & 112.5 & 122.8 & 120.6 & 109.9 & 103.8 & 120.4 & 109.7 & 103.6 \\
\hline$\widehat{\widehat{Y}}_{16}$ & 103.7 & 109.7 & 119.7 & 117.6 & 107.2 & 101.2 & 117.4 & 106.9 & 101.0 \\
\hline$\widehat{\bar{Y}}_{17}$ & 116.7 & 123.4 & 134.6 & 132.3 & 120.5 & 113.8 & 132.1 & 120.3 & 113.6 \\
\hline
\end{tabular}

Table 4: PRE of the proposed estimators and the existing estimators for population 2

\begin{tabular}{|c|c|c|c|c|c|c|c|c|c|}
\hline \multirow{2}{*}{$\begin{array}{l}\text { Existing } \\
\text { Estimators }\end{array}$} & \multicolumn{9}{|c|}{ Proposed Estimators } \\
\hline & $\widehat{\widehat{Y}}_{p 1}$ & $\widehat{\widehat{Y}}_{p 2}$ & $\widehat{\widehat{Y}}_{p 3}$ & $\widehat{\widehat{Y}}_{p 4}$ & $\widehat{\widehat{\boldsymbol{Y}}}_{p 5}$ & $\widehat{\widehat{Y}}_{p 6}$ & $\widehat{\widehat{Y}}_{p 7}$ & $\widehat{\bar{Y}}_{p 8}$ & $\widehat{\widehat{Y}}_{p 9}$ \\
\hline$\widehat{\bar{Y}}_{r}$ & 164.3 & 172.5 & 178.4 & 159.7 & 154.7 & 147.7 & 157.9 & 153.0 & 146.1 \\
\hline$\widehat{\bar{Y}}_{1}$ & 368.5 & 386.9 & 400.1 & 358.1 & 346.8 & 331.2 & 354.1 & 343.0 & 327.6 \\
\hline$\widehat{\widehat{Y}}_{2}$ & 366.1 & 384.4 & 397.5 & 355.8 & 344.6 & 329.0 & 351.8 & 340.8 & 325.5 \\
\hline$\widehat{\widehat{Y}}_{3}$ & 366.8 & 385.0 & 398.2 & 356.4 & 345.2 & 329.6 & 352.4 & 341.4 & 326.1 \\
\hline$\widehat{\widehat{Y}}_{4}$ & 365.1 & 383.3 & 396.4 & 354.8 & 343.6 & 328.1 & 350.8 & 339.8 & 324.6 \\
\hline$\widehat{\widehat{Y}}_{5}$ & 366.7 & 384.9 & 398.1 & 356.3 & 345.1 & 329.5 & 352.3 & 341.3 & 326.0 \\
\hline$\widehat{\widehat{Y}}_{6}$ & 366.2 & 384.5 & 397.6 & 355.9 & 344.7 & 329.1 & 351.9 & 340.9 & 325.6 \\
\hline$\widehat{\widehat{Y}}_{7}$ & 366.1 & 384.3 & 397.5 & 355.7 & 344.5 & 329.0 & 351.8 & 340.8 & 325.5 \\
\hline$\widehat{\widehat{Y}}_{\mathbf{8}}$ & 365.9 & 384.2 & 397.3 & 355.6 & 344.4 & 328.8 & 351.6 & 340.6 & 325.3 \\
\hline$\widehat{\bar{Y}}_{9}$ & 365.2 & 383.4 & 396.6 & 354.9 & 343.7 & 328.2 & 350.9 & 339.9 & 324.7 \\
\hline$\widehat{\bar{Y}}_{10}$ & 366.6 & 384.9 & 398.0 & 356.2 & 345.0 & 329.5 & 352.3 & 341.2 & 325.9 \\
\hline$\widehat{\bar{Y}}_{11}$ & 365.3 & 383.5 & 396.6 & 354.9 & 343.8 & 328.3 & 351.0 & 340.0 & 324.7 \\
\hline$\widehat{\widehat{Y}}_{12}$ & 367.2 & 385.5 & 398.7 & 356.8 & 345.6 & 330.0 & 352.8 & 341.8 & 326.4 \\
\hline$\widehat{\bar{Y}}_{13}$ & 164.3 & 172.5 & 178.4 & 159.7 & 154.7 & 147.7 & 157.9 & 153.0 & 146.1 \\
\hline$\widehat{\widehat{Y}}_{14}$ & 158.7 & 166.6 & 172.3 & 154.2 & 149.4 & 142.6 & 152.5 & 147.7 & 141.1 \\
\hline$\widehat{\bar{Y}}_{15}$ & 192.3 & 201.8 & 208.8 & 186.8 & 180.9 & 172.8 & 184.7 & 179.0 & 170.9 \\
\hline$\widehat{\bar{Y}}_{16}$ & 126.7 & 133.0 & 137.5 & 123.1 & 119.2 & 113.8 & 121.7 & 117.9 & 112.6 \\
\hline$\widehat{\bar{Y}}_{17}$ & 171.9 & 180.4 & 186.6 & 167.0 & 161.8 & 154.5 & 165.1 & 160.0 & 152.8 \\
\hline
\end{tabular}


Table 5: PRE of the proposed estimators and the existing estimators for population 3

\begin{tabular}{|c|c|c|c|c|c|c|c|c|c|}
\hline \multirow{2}{*}{$\begin{array}{c}\text { Existing } \\
\text { Estimators }\end{array}$} & \multicolumn{9}{|c|}{ Proposed Estimators } \\
\hline & $\widehat{\widehat{Y}}_{p 1}$ & $\widehat{\widehat{Y}}_{p 2}$ & $\widehat{\widehat{Y}}_{p 3}$ & $\widehat{\widehat{Y}}_{p 4}$ & $\widehat{\widehat{Y}}_{p 5}$ & $\widehat{\bar{Y}}_{p 6}$ & $\widehat{\widehat{Y}}_{p 7}$ & $\widehat{\widehat{Y}}_{p 8}$ & $\widehat{\widehat{Y}}_{p 9}$ \\
\hline$\hat{\bar{Y}}_{r}$ & 100.4 & 129.1 & 105.6 & 95.6 & 115.7 & 91.2 & 94.6 & 114.4 & 90.3 \\
\hline$\widehat{\widehat{Y}}_{1}$ & 307.8 & 396.0 & 323.7 & 293.2 & 354.8 & 279.6 & 290.2 & 350.9 & 276.9 \\
\hline$\widehat{\widehat{Y}}_{2}$ & 307.4 & 395.5 & 323.3 & 292.8 & 354.4 & 279.2 & 289.9 & 350.4 & 276.5 \\
\hline$\widehat{\widehat{Y}}_{3}$ & 307.8 & 396.0 & 323.8 & 293.2 & 354.9 & 279.6 & 290.3 & 350.9 & 276.9 \\
\hline$\widehat{\widehat{Y}}_{4}$ & 314.2 & 404.2 & 330.5 & 299.3 & 362.2 & 285.4 & 296.3 & 358.2 & 282.6 \\
\hline$\widehat{\bar{Y}}_{5}$ & 307.8 & 396.0 & 323.8 & 293.2 & 354.9 & 279.6 & 290.3 & 351.0 & 276.9 \\
\hline$\widehat{\widehat{Y}}_{6}$ & 307.3 & 395.3 & 323.2 & 292.7 & 354.3 & 279.1 & 289.8 & 350.3 & 276.4 \\
\hline$\widehat{\widehat{Y}}_{7}$ & 307.1 & 395.1 & 323.0 & 292.5 & 354.1 & 278.9 & 289.6 & 350.1 & 276.3 \\
\hline$\widehat{\bar{Y}}_{8}$ & 307.4 & 395.4 & 323.3 & 292.8 & 354.3 & 279.2 & 289.8 & 350.4 & 276.5 \\
\hline$\widehat{\bar{Y}}_{9}$ & 315.9 & 406.4 & 332.2 & 300.9 & 364.1 & 286.9 & 297.9 & 360.1 & 284.1 \\
\hline$\widehat{\widehat{Y}}_{10}$ & 307.8 & 396.0 & 323.8 & 293.2 & 354.9 & 279.6 & 290.3 & 350.9 & 276.9 \\
\hline$\widehat{\widehat{Y}}_{11}$ & 307.2 & 395.3 & 323.2 & 292.6 & 354.2 & 279.0 & 289.7 & 350.3 & 276.4 \\
\hline$\widehat{\widehat{Y}}_{12}$ & 307.8 & 396.0 & 323.8 & 293.2 & 354.9 & 279.6 & 290.3 & 350.9 & 276.9 \\
\hline$\widehat{\widehat{Y}}_{13}$ & 114.9 & 147.9 & 120.9 & 109.5 & 132.5 & 104.4 & 108.4 & 131.0 & 103.4 \\
\hline$\widehat{\widehat{Y}}_{14}$ & 91.1 & 117.2 & 95.9 & 86.8 & 105.1 & 82.8 & 85.9 & 103.9 & 82.0 \\
\hline$\widehat{\widehat{Y}}_{15}$ & 119.2 & 153.3 & 125.3 & 113.5 & 137.4 & 108.2 & 112.4 & 135.9 & 107.2 \\
\hline$\widehat{\widehat{Y}}_{16}$ & 10.9 & 14.0 & 11.4 & 10.3 & 12.5 & 9.9 & 10.2 & 12.4 & 9.8 \\
\hline$\widehat{\bar{Y}}_{17}$ & 141.5 & 182.1 & 148.9 & 134.8 & 163.1 & 128.5 & 133.5 & 161.3 & 127.3 \\
\hline
\end{tabular}

Table 6: PRE of the proposed estimators and the existing estimators for population 4

\begin{tabular}{|c|c|c|c|c|c|c|c|c|c|}
\hline \multirow{2}{*}{$\begin{array}{c}\text { Existing } \\
\text { Estimators }\end{array}$} & \multicolumn{9}{|c|}{ Proposed Estimators } \\
\hline & $\widehat{\widehat{Y}}_{p 1}$ & $\widehat{\widehat{Y}}_{p 2}$ & $\widehat{\widehat{Y}}_{p 3}$ & $\widehat{\widehat{Y}}_{p 4}$ & $\widehat{\widehat{Y}}_{p 5}$ & $\widehat{\widehat{Y}}_{p 6}$ & $\widehat{\widehat{Y}}_{p 7}$ & $\widehat{\widehat{Y}}_{p 8}$ & $\widehat{\widehat{Y}}_{p 9}$ \\
\hline$\widehat{\bar{Y}}_{r}$ & 146.1 & 131.9 & 149.3 & 162.0 & 144.3 & 159.2 & 161.8 & 144.1 & 159.0 \\
\hline$\widehat{\widehat{Y}}_{1}$ & 132.0 & 119.2 & 134.9 & 146.3 & 130.3 & 143.9 & 146.2 & 130.2 & 143.7 \\
\hline$\widehat{\widehat{Y}}_{2}$ & 132.0 & 119.2 & 134.9 & 146.3 & 130.3 & 143.9 & 146.2 & 130.2 & 143.7 \\
\hline$\widehat{\widehat{Y}}_{3}$ & 131.8 & 119.1 & 134.8 & 146.2 & 130.2 & 143.7 & 146.1 & 130.0 & 143.6 \\
\hline$\widehat{\bar{Y}}_{4}$ & 132.0 & 119.2 & 134.9 & 146.3 & 130.3 & 143.9 & 146.2 & 130.2 & 143.7 \\
\hline$\widehat{\widehat{Y}}_{5}$ & 131.9 & 119.2 & 134.8 & 146.3 & 130.3 & 143.8 & 146.1 & 130.1 & 143.6 \\
\hline$\widehat{\widehat{Y}}_{6}$ & 132.0 & 119.2 & 134.9 & 146.3 & 130.3 & 143.9 & 146.2 & 130.2 & 143.7 \\
\hline$\widehat{\widehat{Y}}_{7}$ & 132.0 & 119.2 & 134.9 & 146.3 & 130.3 & 143.9 & 146.2 & 130.2 & 143.7 \\
\hline$\widehat{\widehat{Y}}_{8}$ & 132.0 & 119.2 & 134.9 & 146.3 & 130.3 & 143.9 & 146.2 & 130.2 & 143.7 \\
\hline$\widehat{\widehat{Y}}_{9}$ & 132.0 & 119.2 & 134.9 & 146.3 & 130.3 & 143.9 & 146.2 & 130.2 & 143.7 \\
\hline$\widehat{\widehat{Y}}_{10}$ & 131.8 & 119.1 & 134.7 & 146.2 & 130.2 & 143.7 & 146.0 & 130.0 & 143.5 \\
\hline$\widehat{\widehat{Y}}_{11}$ & 131.9 & 119.2 & 134.9 & 146.3 & 130.3 & 143.8 & 146.2 & 130.1 & 143.7 \\
\hline$\widehat{\widehat{Y}}_{12}$ & 131.9 & 119.2 & 134.9 & 146.3 & 130.3 & 143.8 & 146.2 & 130.1 & 143.7 \\
\hline$\widehat{\widehat{Y}}_{13}$ & 113.3 & 102.3 & 115.8 & 125.6 & 111.9 & 123.5 & 125.5 & 111.7 & 123.4 \\
\hline$\widehat{\widehat{Y}}_{14}$ & 121.4 & 109.7 & 124.1 & 134.6 & 119.9 & 132.4 & 134.5 & 119.8 & 132.2 \\
\hline$\widehat{\widehat{Y}}_{15}$ & 127.2 & 114.9 & 130.0 & 141.0 & 125.6 & 138.7 & 140.9 & 125.4 & 138.5 \\
\hline$\widehat{\widehat{Y}}_{16}$ & 131.2 & 118.5 & 134.1 & 145.5 & 129.6 & 143.0 & 145.4 & 129.4 & 142.9 \\
\hline$\widehat{\widehat{Y}}_{17}$ & 124.4 & 112.4 & 127.1 & 137.9 & 122.8 & 135.6 & 137.8 & 122.7 & 135.4 \\
\hline
\end{tabular}


The proposed estimators perform well as compared to the usual ratio estimator and the existing estimators in terms of PRE (cf. Tables 3-6). This shows that the suggested ratio type estimators are more efficient than the usual ratio estimator and the existing ratio estimators.

\section{Conclusion}

Availability of the auxiliary information is helpful to improve the sampling plan or to enhance the estimation of the properties of the variables of interest. Some new modified linear regression type ratio estimators using known values of the population coefficient of variation, population correlation coefficient, the Gini's mean difference, Downton's method and probability weighted moments based measures are suggested. The proposed estimators are more efficient than that of the existing estimators in terms of constant, bias and mean square error under different populations. Hence we strongly recommend the use of the proposed modified linear regression type ratio estimators for practical concerns and the estimation of the population mean and may be preferred over the usual ratio estimator and the existing modified linear regression type ratio estimators when unusual observations are present in the auxiliary variables.

\section{References}

1. Cochran, W. G. (1940). Sampling Techniques, Third Edition, Wiley Eastern Limited.

2. Jeelani, M. I. Maqbool, S. and Mir, S. A. (2013). Modified ratio estimators of population mean using linear combination of coefficient of skewness and quartile deviation. International Journal of Modern Mathematical Sciences, (6), 174-183.

3. Kadilar, C. and Cingi, H. (2004). Ratio estimators in simple random sampling. Applied Mathematics and Computation, 151, 893-902.

4. Kadilar, C. and Cingi, H. (2006). An Improvement in estimating the population mean by using the correlation coefficient, Hacettepe Journal of Mathematics and Statistics, 35 (1), 103-109.

5. Murthy, M.N. (1967). Sampling Theory and Methods. Statistical Publishing Society, Calcutta, India.

6. Rao, T.J. (1991). On certain methods of improving ratio and regression estimators. Communications in Statistics-Theory and Methods, 20 (10), 33253340 .

7. Singh, D. and Chaudhary, F.S. (1986). Theory and Analysis of Sample Survey Designs. New Age International Publisher.

8. Singh, H.P. and Tailor, R. (2003). Use of known correlation coefficient in estimating the finite population means, Statistics in Transition, 6 (4), 555-560.

9. Singh, H. P., Tailor, R., Tailor, R. and Kakran, M.S. (2004). An improved estimator of population mean using power transformation. Journal of the Indian Society of Agricultural Statistics, (58), 223-230. 
10. Sisodia, B.V.S. and Dwivedi, V.K. (1981). A modified ratio estimator using coefficient of variation of auxiliary variable. Journal of the Indian Society of Agricultural Statistics, 33(1), 13-18.

11. Subramani, J. and Kumarapandiyan, G. (2012a). Estimation of Population Mean using Co-efficient of variation and Median of an Auxiliary Variable. International Journal of Probability and Statistics, (1), 111-118.

12. Subramani, J. and Kumarapandiyan, G. (2012b). Estimation of population mean using known median and co-efficient of skewness. American Journal of Mathematics and Statistics, (2), 101-107.

13. Subramani, J. and Kumarapandiyan, G. (2012c). Modified ratio estimators using known median and co-efficient of kurtosis. American Journal of Mathematics and Statistics, (2), 95-100.

14. Subramani, J.and Kumarapandiyan, G. (2102d). A class of modified ratio estimators using Deciles of an Auxiliary Variable. International Journal of Statistical Application, 2, 101-107.

15. Upadhyaya, L.N. and Singh, H.P. (1999): Use of transformed auxiliary variable in estimating the finite population mean, Biometrical Journal, 41(5), 627-636.

16. Yan, Z. and Tian, B. (2010): Ratio method to the mean estimation using coefficient of skewness of auxiliary variable, ICICA 2010, Part II, CCIS 106, 103-110. 